\title{
COUPLED BOUNDARY ELEMENT: STOCHASTIC COLLOCATION APPROACH FOR THE UNCERTAINTY ESTIMATION OF SIMULATIONS OF TRANSCRANIAL ELECTRIC STIMULATION
}

\author{
JURE RAVNIK ${ }^{1}$, ANNA ŠUŠNJARA ${ }^{2}$, OŽBEJ VERHNJAK ${ }^{1}$, DRAGAN POLJAK ${ }^{2} \&$ MARIO CVETKOVIĆ $^{2}$ \\ ${ }^{1}$ Faculty of Mechanical Engineering, University of Maribor, Slovenia \\ ${ }^{2}$ Faculty of Electrical Engineering, Mechanical Engineering and Naval Architecture, University of Split, Croatia
}

\begin{abstract}
In this paper, the authors present a deterministic model of transcranial electric stimulation and develop a Boundary Element Method based algorithm capable of calculating potential and current density in the investigated domain. Furthermore, the deterministic model is coupled with Stochastic Collocation Method to evaluate the propagation of the uncertainty of the input parameters to the results. The uncertainty of the results is analysed via statistical approaches. The governing partial differential equation of the deterministic model is the Laplace equation. We assume that the studied domain is composed of subdomains of different materials. The subdomains are homogeneous and isotropic and have constant electrical conductivity. We introduce the Boundary Element Method for such a setup, support Dirichlet and Neumann boundary conditions at the outer boundary of the domain, and assume continuity of the potential and conservation of current density at the boundaries between the subdomains. We assume that the electrical conductivity of each subdomain is subject to some uncertainty. By coupling the developed simulation tool with the Stochastic Collocation Method, we are able to analyse the uncertainty of the resulting electric potential and current density and identify the contribution to the uncertainty from each subdomain. We apply the developed algorithms to study the transcranial electric stimulation of a human head. A head model with 9 tissues (white and grey matter parts of cerebellum, ventricles, cerebellum, cerebrospinal fluid, head, tongue, cerebrum, and skull) is considered and voltage is applied across two electrodes. The results show that the uncertainty of electrical conductivity in the skull, cerebrospinal fluid and, grey matter have the largest influence on the results.

Keywords: boundary element method, stochastic collocation method, sensitivity analysis, transcranial electric stimulation.
\end{abstract}

\section{INTRODUCTION}

The Boundary Element Method (BEM) is a numerical technique aimed at solving partial differential equations (PDEs). In the case of a homogenous PDE, using its fundamental solution, we derive a boundary integral equation, which enables us to find the solution by discretizing only the boundary of the problem domain. The authors consider the modelling of Transcranial Electric Stimulation (TES) in this paper. Transcranial electrical stimulation (TES) represents a non-invasive brain stimulation technique used as a treatment in various brain related disorders, as well as an important tool in stroke recovery and chronic pain [1]. Crucial to the application of TES is the knowledge on the conductivity of biological tissues. However, mostly due to a different measurement methods and tissue preparation techniques, but also due to particularly challenging measurement in the low frequency range, there is a high level of discrepancy between the reported values of tissue properties. These tissue uncertainties are considered a main concern in computational models of TES since tissue conductivity values will have a significant impact on the distribution of the induced fields [1]. Therefore, we consider the coupling of deterministic TES model with statistical methods to take into account this implicit uncertainty. TES, for a homogenous domain, is governed by 
the Laplace equation. When applied to the stimulation of the human head, inhomogeneous conditions as the electrical conductivity of different tissues varies are encountered. Domain decomposition (Bui et al. [2]) is considered to handle different conductivities of the different tissues.

The main goal of this work is to couple the deterministic BEM based simulation tool with the Stochastic Collocation Method (SCM, Babuska et al. [3]) to estimate the uncertainty of the numerical predictions. Numerical modelling and simulation are advantageous in many cases to performing experimental measurements and are thus widely used in almost all engineering disciplines for design and optimization purposes. The use of a purely deterministic model and a BEM solution leads to simulation results, but does not contain any information about the uncertainty of the results. Such an estimation can be obtained by comparing simulation results with experiments, but these are costly and time consuming. The SCM allows the propagation of the uncertainty of input parameters (such as tissue conductivity in the case of TES) through the numerical model to the results (such as electric potential or current in the case of TES).

\section{GOVERNING EQUATIONS}

To study bioelectromagnetism in living tissues, the authors consider them as volume conductors [4]. Their resistances, capacitances, and voltage sources are distributed within a volume, and the inductive component of the impedance is neglected. For low frequencies, we can neglect capacitive and electromagnetic effects. Thus, currents and voltages can be considered stationary within this quasi-static approximation.

Let us consider a tissue as a conductor with electrical conductivity $\sigma$ in which there are no volumetric current sources. If an electric field $\vec{E}$ is assumed, the net current flow into/out of the volume must be solenoidal, i.e.

$$
\vec{\nabla} \cdot \vec{J}=0
$$

where $\vec{J}$ is the current density. It is related to the electric field via $\vec{J}=\sigma \vec{E}$. In static conditions, the electric field may be expressed as a negative gradient of the electric potential $\varphi, \vec{E}=-\vec{\nabla} \varphi$. With this, we may rewrite eqn (1) as

$$
\vec{\nabla} \cdot(-\sigma \vec{\nabla} \varphi)=0
$$

In general, the electrical conductivity for an anisotropic conductor is a tensor. For example, in the white matter of the brain, the electrical conductivity is higher in the direction of the nerve fiber tracts. In this work, we model the tissues in the human head as a group of subdomains, each representing individual tissues with different but homogeneous and isotropic electrical conductivity. In this case, the eqn (2) for each subdomain simplifies to a Laplace equation

$$
\nabla^{2} \varphi=0
$$

where changes of electric conductivity between tissues are taken into account through boundary conditions. At the outer boundary of the computational domain we prescribe either Dirichlet boundary condition (known electric potential $\varphi$ ) or Neumann boundary conditions (known projection of electric current density to normal direction $J=-\sigma \vec{n} \cdot \vec{\nabla} \varphi$ ). Here, $\vec{n}$ is the outward pointing unit normal at the boundary. At the boundaries between subdomains continuity of potential $\left(\varphi_{I}=\varphi_{I I}\right)$ and conservation of current density $\left(J_{I}=-J_{I I}\right)$ is prescribed. 


\section{NUMERICAL ALGORITHM}

\subsection{The boundary element method for TES}

A three-dimensional domain $\Omega \in \mathbb{R}^{3}$ with a boundary $\Gamma=\partial \Omega$ and a location vector $\vec{r}$ is considered. The domain is locally homogeneous, so different sub-regions have different, but constant material properties. The authors employ the domain-decomposition approach and divide the domain into subdomains $\Omega=\cup \Omega_{i}$, where each subdomain has its own boundary $\Gamma_{i}=\partial \Omega_{i}$. Inside each subdomain we can write a boundary integral representation of the Laplace eqn (3) as [5]

$$
c(\vec{\xi}) \varphi(\vec{\xi})+\int_{\Gamma_{i}} \varphi(\vec{r}) \vec{\nabla} \varphi^{\star} \cdot \vec{n} d \Gamma_{i}=\int_{\Gamma_{i}} \varphi^{\star}(\vec{n} \cdot \vec{\nabla} \varphi(\vec{r})) d \Gamma_{i} \quad \vec{\xi} \in \Gamma_{i},
$$

where $\Gamma_{i}$ is the boundary of $i^{t h}$ subdomain, $\vec{\xi}$ is the source point, $c$ is the free coefficient, and $\varphi^{\star}=1 / 4 \pi|\vec{r}-\vec{\xi}|$ is the fundamental solution of the Laplace operator. Such a representation allows us to solve only for the unknowns at the boundary of the subdomain, since the solution in the interior depends only on the knowledge of boundary variables (potential $\varphi(\vec{r})$ and flux $q=\vec{n} \cdot \vec{\nabla} \varphi(\vec{r}))$.

To obtain a system of linear equations for the unknowns at the boundary, we discretize subdomain boundaries with triangular elements. Within the triangles we use a linear interpolation of the potential $\varphi(\vec{r})=\sum \Phi_{j} \varphi(\vec{r})_{j}$ and a constant interpolation of the flux. The collocation point is placed into each interpolation vertex (the three corners and the barycentre of each boundary element). The two integrals in eqn (4) are evaluated and stored into matrices $\left[H_{i}\right]$ and $\left[G_{i}\right]$. Each row of the matrix corresponds to a collocation point and each column to a node in the computational mesh. The matrix entries are

$$
\left[H_{i}^{(j k)}\right]=\int_{\Gamma_{i}^{(k)}} \Phi_{k} \vec{\nabla} \varphi_{j}^{\star} \cdot \vec{n} d \Gamma, \quad\left[G_{i}^{(j k)}\right]=\int_{\Gamma_{i}^{(k)}} \varphi_{j}^{\star} d \Gamma .
$$

where $i$ denotes the subdomain, $\Gamma_{i}^{(k)}$ is the boundary element, $j$ the collocation point and $k$ the column index corresponding to the node index of the chosen boundary element and interpolation shape function. Contribution to a node $k$ from different adjacent boundary elements, which share the node $k$ are summed up and stored in a single matrix entry. Using the matrices of integrals we obtain the following system of linear equations for each subdomain

$$
c_{i} \varphi_{i}+\left[H_{i}\right]\{\varphi\}=\left[G_{i}\right]\{q\},
$$

where $\{\varphi\}$ and $\{q\}$ are vectors of nodal values of potential and flux. The computation of the free coefficient $c(\vec{\xi})$ and the strongly singular diagonal element of $\left[H_{i}\right]$ is done indirectly. Setting $\varphi=1, q=0$ as one of the valid solutions of the original problem, we can use eqn (6) to evaluate the sum of $c$ and the diagonal element in the $\left[H_{i}\right]$ matrix if all other elements were previously evaluated by numerical integration.

Integrals in eqn (5) are performed numerically over triangles in 3D space. For the purpose of linear interpolation within each triangle it is convenient to use barycentric coordinate system $\left(\lambda_{1}, \lambda_{2}, \lambda_{3}\right)$. A position within a triangle is then calculated by $\vec{r}=\lambda_{1} \vec{r}_{1}+\lambda_{2} \vec{r}_{2}+$ $\lambda_{3} \vec{r}_{3}$. Weights and integration point locations were taken out of [6] and converted to the barycentric coordinate system. Lists of $\left(w_{i}, \lambda_{1, i}, \lambda_{2, i}, \lambda_{3, i}\right)$ with 7, 25, 54, 85 and 126 entries were obtained, which provide machine precision accurate integration of polynomials of degree 5, 10, 15, 20 and 25 respectively. With this, integral over a triangular boundary element 
$\tau$ can be approximated by

$$
\int_{\tau} f(\vec{r}) d \Gamma \approx \sum_{i} w_{i} f\left(\lambda_{1, i} \vec{r}_{1}+\lambda_{2, i} \vec{r}_{2}+\lambda_{3, i} \vec{r}_{3}\right) .
$$

When applying the boundary element method singular integrals must be computed. The singularity is located at one of the vertices of the triangle (in linear interpolation methods) or at the centre of the triangle (in constant interpolation methods). To accurately compute such integrals, we recursively subdivide the triangle in the direction of the singular point. Fig. 1 demonstrates the recursive algorithm. At each step, the triangle is subdivided into four smaller triangles obtained by bisecting the sides of the triangle. The one small triangle that still contains the singularity is subdivided again in the next step. The number of parts into which the triangle is divided is $1+3 n$, where $n$ is the number of recursive steps. The final result is a sum of integrals calculated over all the parts. The number of recursive steps controls the accuracy and can be adjusted to match the accuracy of computing non-singular integrals.

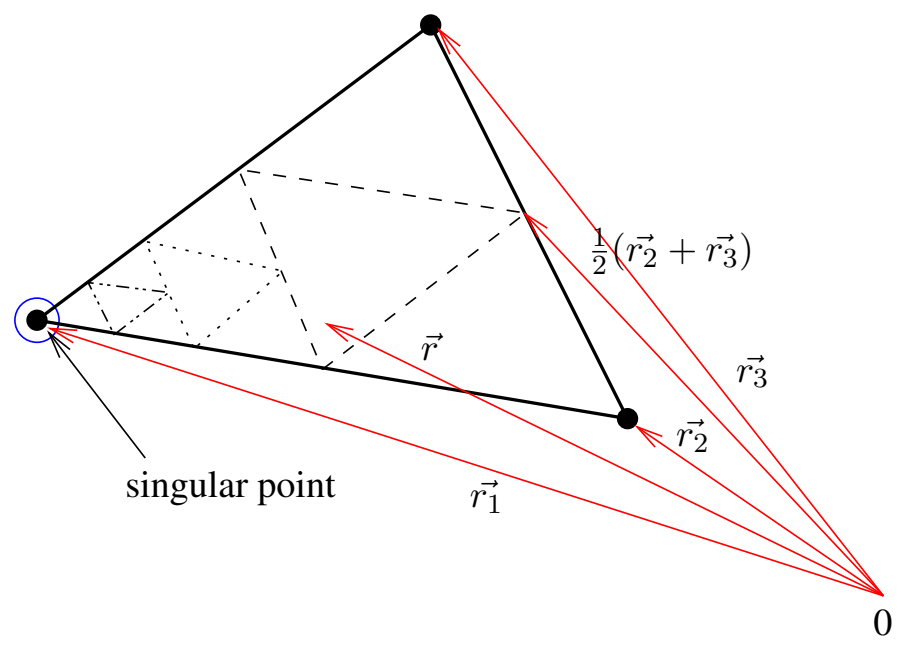

Figure 1: Recursive subdivision of a triangle element towards the singular point.

Having set up the systems of linear equations (eqn (6)) for each subdomain, let us examine them and discuss the boundary conditions. The number of equations (rows in matrices) in these systems is equal to the number of all nodes in the mesh. This includes all vertices of all triangles and all barycenters. For each boundary element located at the outer boundary, either the potential (at the triangle vertices) or the flux (at the barycenter) is known. At parts of the boundary, which are adjacent to neighboring subdomains, the potential and flux are unknown. Such a system is underdetermined and cannot be solved alone. To mitigate this, continuity of potential and conservation of current density at the boundaries between subdomains is assumed. This gives us an overdetermined system of linear equations (since the nodes are shared between subdomains). The resulting overdetermined linear system of equations is solved for the unknown potential and flux at outer and inner boundary using a least squares based solver (Paige and Saunders [7]). An alternative approach was proposed by Loeffler and Mansur [8], who use a domain superposition instead of a domain decomposition. After the solution at the boundaries has been found, it is possible to acquire the value of potential at any 
point in the domain explicitly by using eqn (4) and setting the source point $\vec{\xi}$ at the desired location.

\subsection{Numerical code verification}

To verify the numerical algorithm consider a 1D potential distribution problem within three joined subdomains. The domain is a long cylinder of diameter $1 \mathrm{~m}$ and length $3 \mathrm{~m}$, as shown in Fig. 2. The prescribed potential is at the top and bottom of the cylinder is $\varphi_{0}=1 \mathrm{~V}$, $\varphi_{3}=4 \mathrm{~V}$ and the conductivities are $\sigma_{1}=2 \mathrm{~S} / \mathrm{m}, \sigma_{2}=3 \mathrm{~S} / \mathrm{m}, \sigma_{3}=4 \mathrm{~S} / \mathrm{m}$. There is no current at the cylinder wall, so the problem is one-dimensional. The analytical solution for the potential between the subdomains is $\varphi_{1}=34 / 13 \mathrm{~V}, \varphi_{2}=66 / 39 \mathrm{~V}$ and for the current density is $J=36 / 13 \mathrm{~A} / \mathrm{m}^{2}$. Several discretizations of the domain were prepared and the results were compared with analytical values. Relative error is used to present the results in Fig. 3. We observe the developed algorithm is second order accurate for current density and even better for potential. At accuracy below $10^{-6}$ convergence stops due to the finite accuracy of numerical integration. In absolute sense the accuracy of potential is better than the accuracy of current density due to the fact we use linear interpolation of potential over boundary elements and only constant interpolation of current density within the boundary elements.

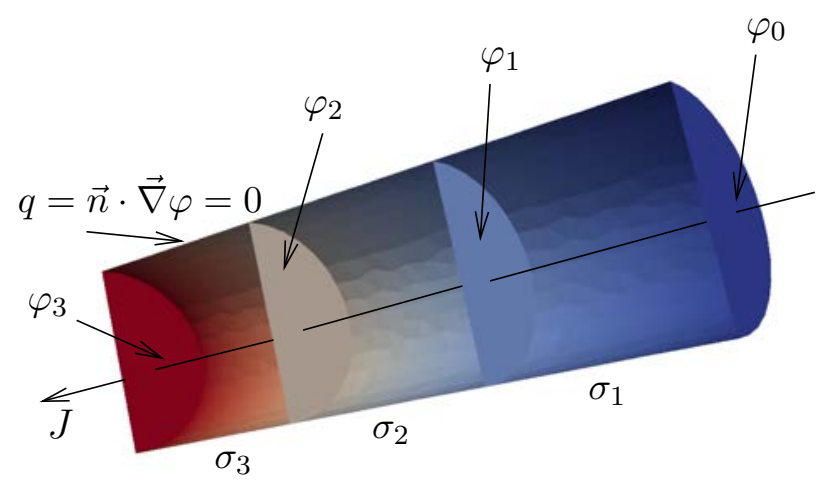

Figure 2: Potential distribution in the case of one-dimensional electrostatics problem with three subdomains. Constant potential $\varphi_{0}$ and $\varphi_{3}$ are prescribed at the top and bottom ends of the cylinder, whereas zero flux is assumed at the cylinder surface.

\section{STOCHASTIC COLLOCATION METHOD}

We consider the conductivity of the tissues in head model to be random variables uniformly distributed in a range $\sigma \in\left(\sigma_{\min }, \sigma_{\max }\right)$. The size of this interval is due to changes between individuals, which was encountered when measuring samples of brain tissue from different donors. We assume uniform distribution, so probability distribution function (PDF) of all random variables is non-zero only in this range: $\left(\sigma_{\min }, \sigma_{\max }\right)$ :

$$
p(\sigma)=\left\{\begin{array}{cc}
\frac{1}{\sigma_{\max }-\sigma_{\min }} & \sigma \in\left(\sigma_{\min }, \sigma_{\max }\right) . \\
0 & \text { elsewhere }
\end{array}\right.
$$

Let the number of random variables (different tissue conductivities in our model) be $n$. Additionally let our deterministic model, which solves for the potential in the head and 


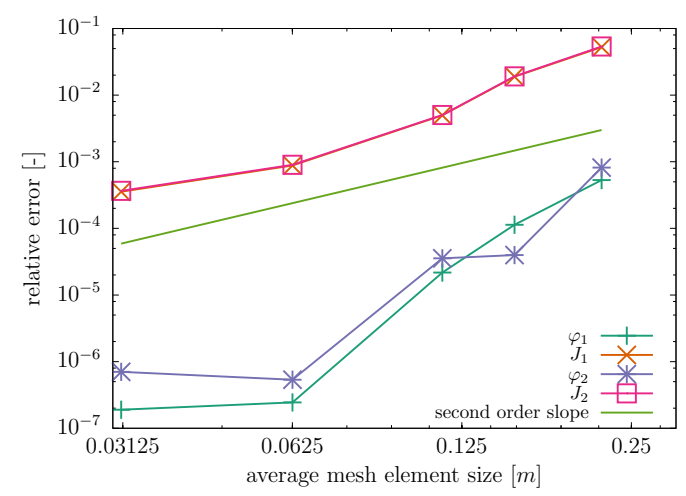

Figure 3: Relative error in potential and current density calculated as the difference between analytical and numerical solution at interfaces between subdomain. The green line shows the second order convergence slope. Average mesh element size is calculated as a square root of average mesh element area.

depends on the conductivities, be denoted by $y\left(\sigma_{1}, \ldots, \sigma_{n}\right)$. In this case, statistics for our deterministic model such as expected value $\mu_{y}$ and variance $v a r_{y}$ may be calculated using [9]:

$$
\begin{gathered}
Y_{i}=\int_{-\infty}^{\infty} \ldots \int_{-\infty}^{\infty}\left[y\left(\sigma_{1}, \ldots, \sigma_{n}\right)\right]^{i} p\left(\sigma_{1}\right) \ldots p\left(\sigma_{n}\right) d \sigma_{1} \ldots d \sigma_{n} \\
\mu_{y}=Y_{1}, \quad \operatorname{var}_{y}=Y_{2}-\mu_{y}^{2} .
\end{gathered}
$$

Evaluating the integral (9) using a standard approach such as Gauss-Lengendre quadrature is possible, however it is very CPU intensive, as the number of evaluations of the model $y$ scales as $N^{n}$, where $N$ is the number of quadrature sample points. To avoid this, we use the Smolyak [10]- [12] sparse grid approach to numerically evaluate the integral (9). The integral is approximated by

$$
Y_{i} \approx \frac{1}{2^{n}} \sum_{i=1}^{N_{s}}\left[y\left(\xi_{i}^{(1)}, \xi_{i}^{(2)}, \ldots, \xi_{i}^{(n)}\right)\right]^{i} w_{i},
$$

where $\xi_{i}^{(t)}=\sigma_{t, \min }+\left(\sigma_{t, \max }-\sigma_{t, \min }\right) \frac{\eta_{i}+1}{2}$ and $\eta_{i}$ and $w_{i}$ are sparse grid points and weights and the $\frac{1}{2^{n}}$ factor comes as a results of change of variables. For large $n$ the number of sparse grid points in eqn (11) and with this the number of evaluations of the deterministic model is much smaller than the number of points needed by standard approaches, such as the Gauss-Lengendre quadrature, i.e. $N_{s} \ll N^{n}$. To calculate the points and weights for the sparse grid we employed the Tasmanian library [13], [14] using Clenshaw Curtis fully nested points.

\section{RESULTS}

We apply the developed method to a human head model with nine tissues. The model and the considered tissues are shown in Fig. 4. Since the ventricles are filled with cerebrospinal fluid, we consider the conductivity of these two subregions as one parameter. The indeterminacy of the conductivity in the jaw was neglected because it has several orders of magnitude less 
influence on the results compared to the other conductivities. Thus, a total of 7 conductivities are considered as random variables: Cerebellum, CSF, grey matter, head, tongue, cerebrum and skull. The potential at the electrodes was set to $\pm 1 \mathrm{~V}$. Zero current boundary condition was used at the outer surface of the head.

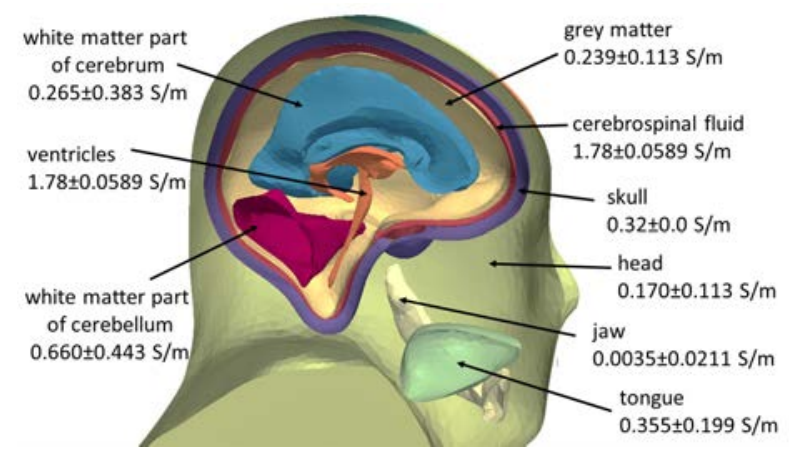

Figure 4: The nine tissue head model, with subdomains representing ventricles, white matter parts of cerebrum and cerebellum, grey matter, cerebrospinal fluid, skull, jaw and tongue. All tissues are enclosed into a domain named head, which represents all other tissues in the human head. Conductivity values are obtained from the tissue properties database [15]. Ventricles are filled with cerebrospinal fluid, thus the conductivity of these two subdomains is treated as one parameter.
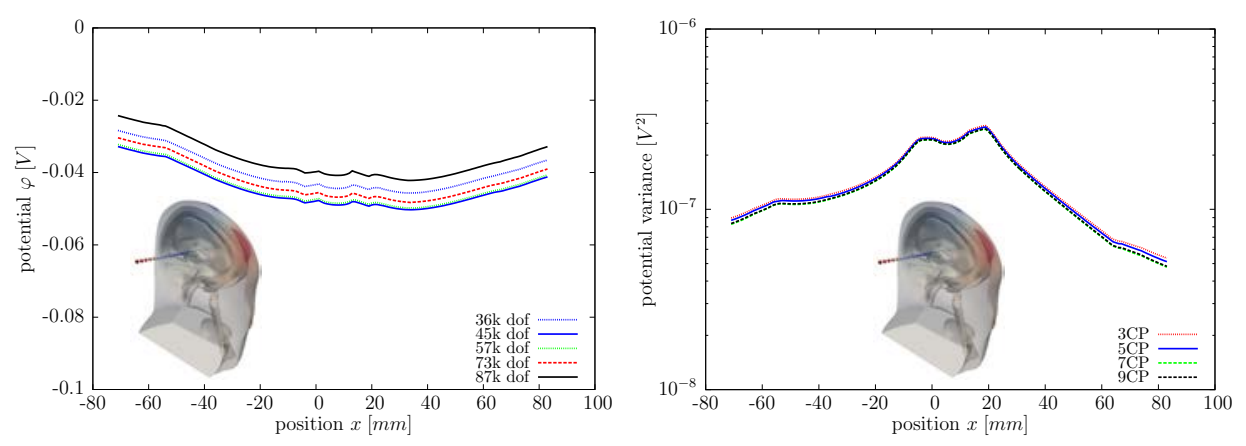

Figure 5: Comparison of potential at left-to-right profile through the head model. Left: Difference in potential due to computational grid density in BEM. Right: Difference in potential variance due to difference in the number of collocation points in SCM. Only small differences between meshes and the number of collocation points are observed.

Different computational grids were constructed with 38,000 to 87,000 degrees of freedom. Only small differences in the results were observed (Fig. 5) so in the interest of saving computational time, we chose the grid with 45,000 degrees of freedom for the analysis. Furthermore, we used SCM with 3, 5, 7 and 9 collocation points to make sure, that the numerical calculation of the integral (9) converges. Comparison in potential variance is 
shown in Fig. 5 and reveals good agreement between results and proves that the numerical calculation of the integral converged. Finally, we chose Smolyak sparse grid approach with 589 simulations to be performed. The total CPU time on a single processor used was about 5 hours per simulation, in total 123 days.

In Fig. 6 we plot the variance of the scalar electric potential $\left[\mathrm{V}^{2}\right]$ at the surfaces of the different tissues in the head. Overall, the largest variance $\approx 10^{-3} \mathrm{~V}^{2}$ is observed in the white matter, grey matter, CFS, skull and head. It makes sense for the CSF to have the large variance due to high conductivity. It is considered a so called "super highway" for current flow, according to Bikson et al. [16]. The greatest variance is observed in the area under the electrodes. This analysis provides information about the uncertainty of the applied electric field to different tissues due to the unknown structure and properties of the tissues.
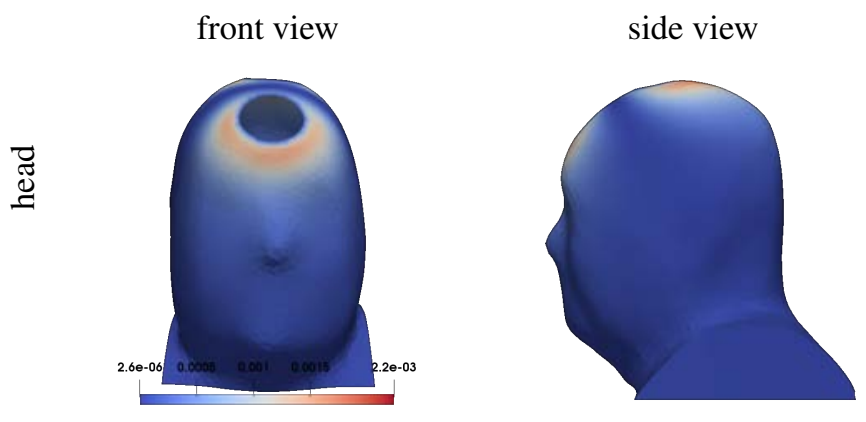

top view
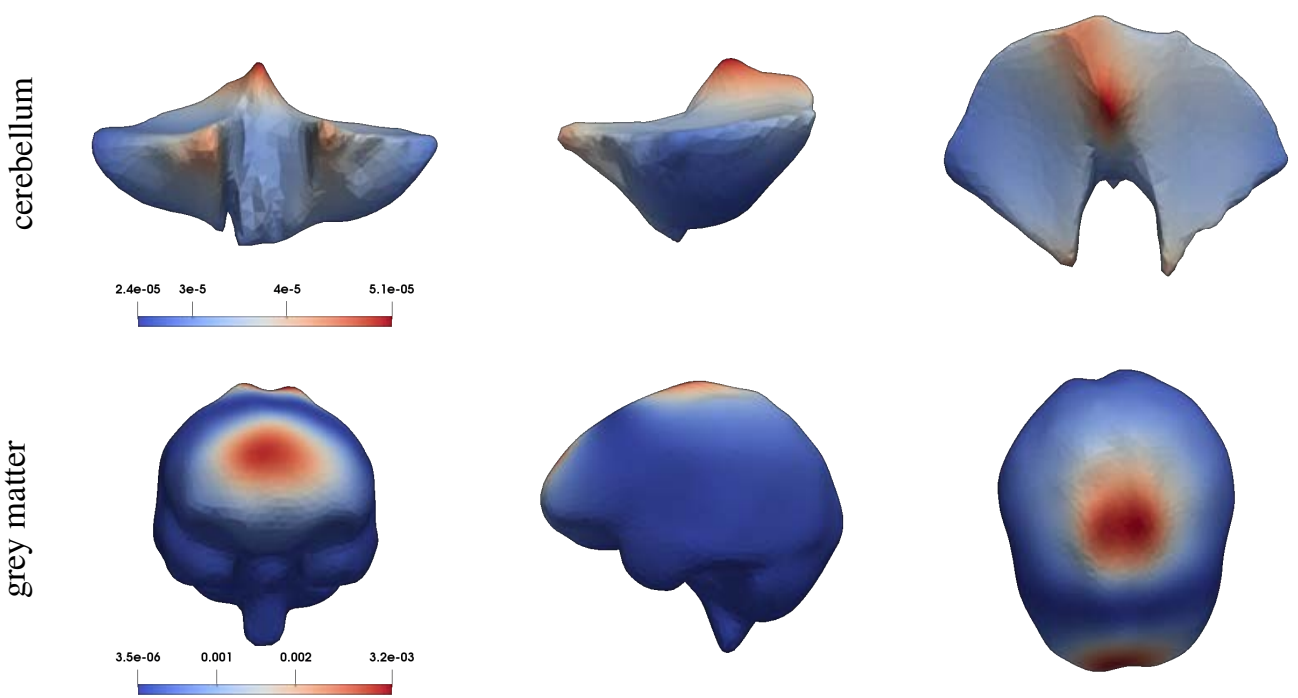

Figure 6: Variance of scalar electric potential $\left[\mathrm{V}^{2}\right]$ shown at surfaces of different tissues in the head. 
front view
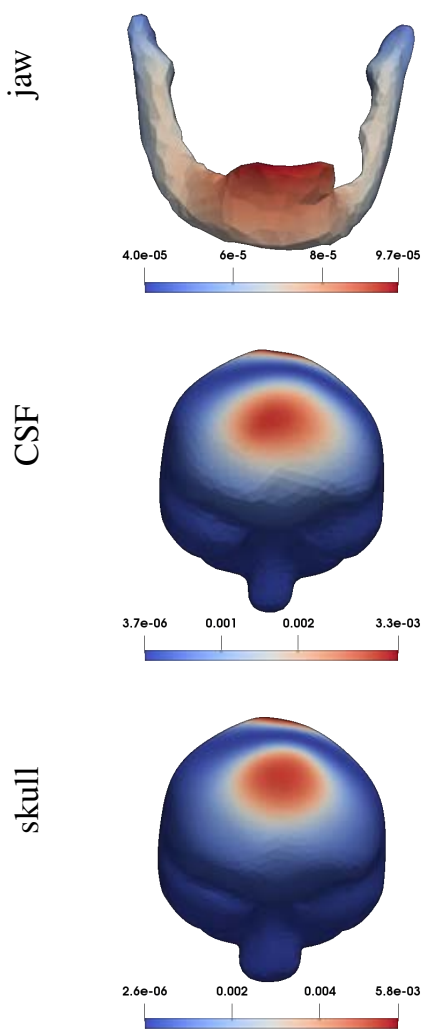

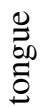

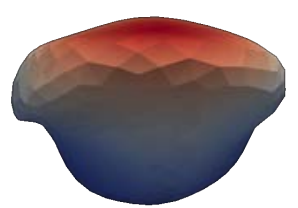

$\begin{array}{ccccc}5.6 e-05 & 8 \mathrm{e}-5 & 0.0001 & 0.00012 & 1.4 \mathrm{e}-04\end{array}$

$\frac{0}{0}$

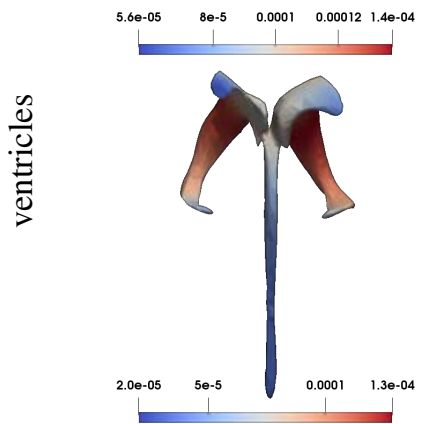

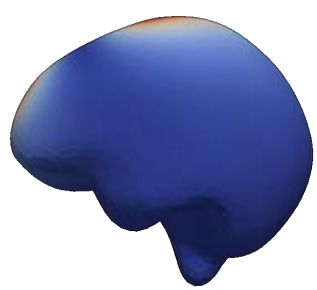
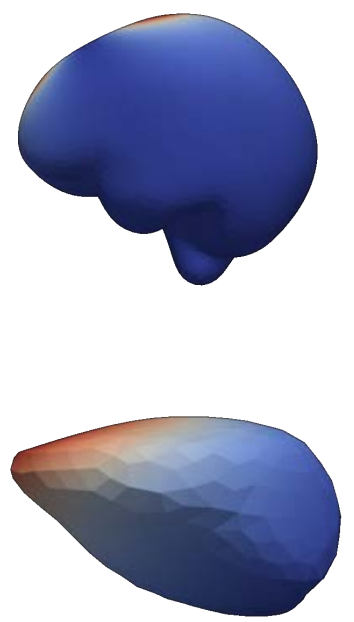

side view

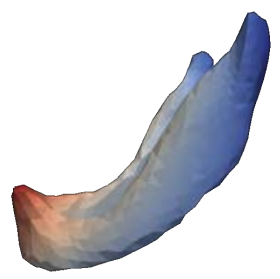

top view
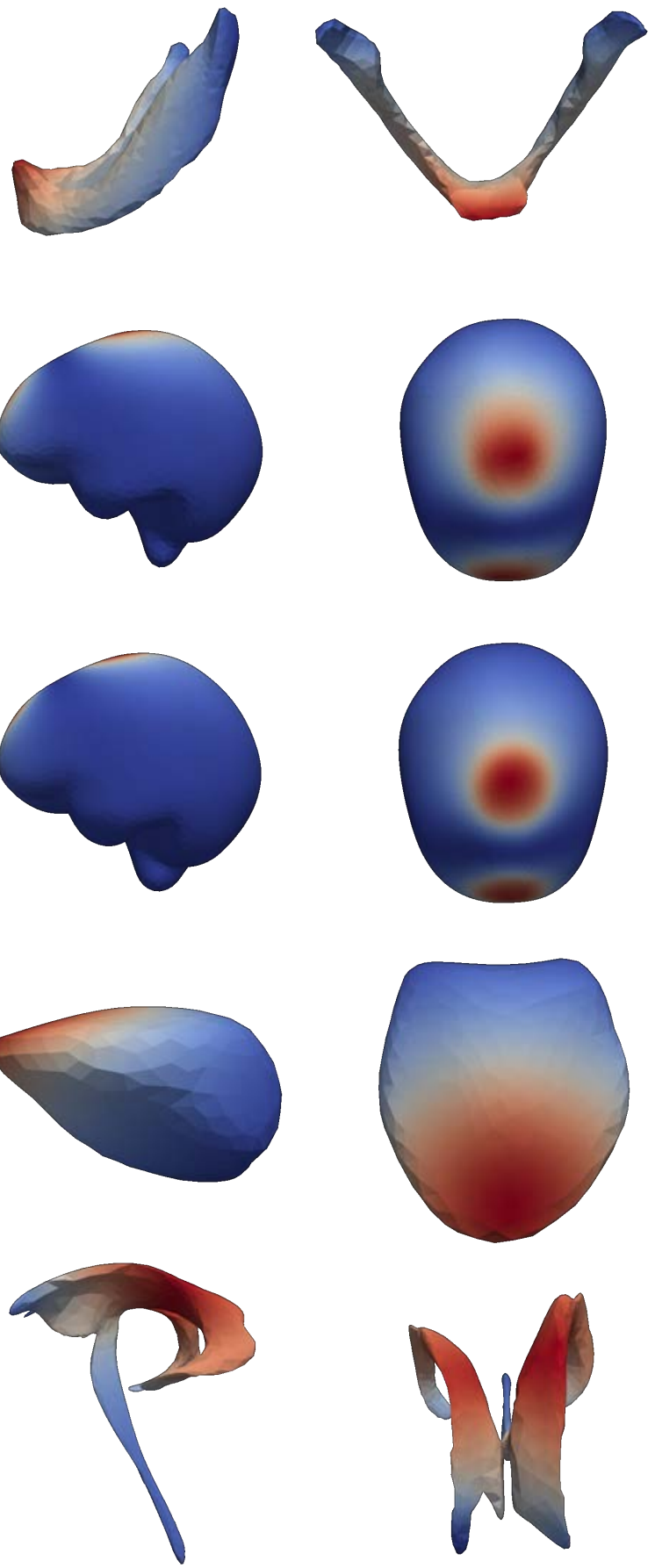

Figure 6: Continued. 

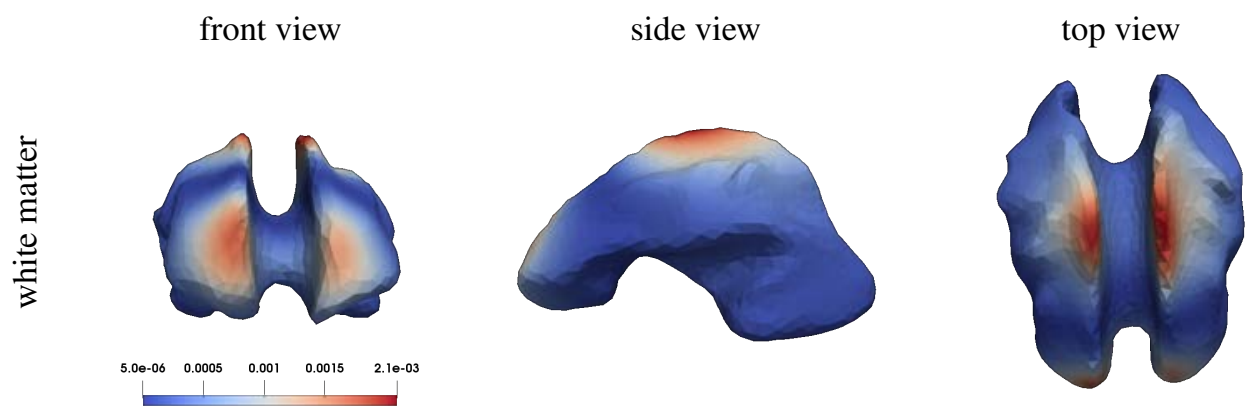

Figure 6: Continued.

\section{CONCLUSIONS}

In this paper, we introduce the Boundary Element Method to simulate the Transcranial Electric Stimulation and couple it with the Stochastic Collocation Method. BEM is a deterministic solver and the SCM wraps it to evaluate the uncertainty quantification of the output of interest. A domain decomposition technique is proposed to deal with changes in material properties in different regions of the model. The domain decomposition technique employed leads to a solution of an overdetermined system of linear equations. The SCM was implemented using a sparse numerical integration scheme, which greatly reduces the number of required deterministic simulations and makes such analyses possible for complex geometries.

The developed method is used to study the TES of a human head model with 9 tissues. The tissues are assumed to be homogeneous and isotropic with constant conductivities. The conductivities of the tissues are considered as random variables uniformly distributed in ranges obtained from the available literature. The expected value and variance values of the electrical scalar potential in the head tissues are calculated. This analysis reveals areas in the human head where the uncertainty of the applied electrical potential is greatest during the TES procedure. We show that the areas directly under the electrodes have the largest variance of the potential. Large variance is observed in white matter, grey matter, cerebrospinal fluid, skull and head.

\section{REFERENCES}

[1] Reato, D., Salvador, R., Bikson, M., Opitz, A., Dmochowski, J. \& Miranda, P.C., Principles of Transcranial Direct Current Stimulation (tDCS): Introduction to the Biophysics of tDCS, Springer International Publishing: Cham, pp. 45-80, 2019.

[2] Bui, T.T. \& Popov, V., Domain decomposition boundary element method with overlapping sub-domains. Engineering Analysis with Boundary Elements, 33(4), pp. 456-466, 2009.

[3] Babuska, I., Nobile, F. \& Tempone, R., A stochastic collocation method for elliptic partial differential equations with random input data. SIAM Review, 52(2), pp. 317-355, 2010.

[4] Bai, S., Loo, C. \& Dokos, S., A review of computational models of transcranial electrical stimulation. Critical Reviews in Biomedical Engineering, 41(1), pp. 21-35, 2013.

[5] Wrobel, L.C., The Boundary Element Method, John Willey \& Sons, Ltd, 2002.

[6] Wandzurat, S. \& Xiao, H., Symmetric quadrature rules on a triangle. Computers and Mathematics with Applications, 45(12), pp. 1829-1840, 2003. 
[7] Paige, C.C. \& Saunders, M.A., LSQR: An algorithm for sparse linear equations and sparse least squares. ACM Transactions on Mathematical Software, 8, pp. 43-71, 1982.

[8] Loeffler, C.F. \& Mansur, W.J., Sub-regions without subdomain partition with boundary elements. Engineering Analysis with Boundary Elements, 71, pp. 169-173, 2016.

[9] Šušnjara, A., Verhnjak, O., Poljak, D., Cvetković, M. \& Ravnik, J., Stochasticdeterministic boundary element modelling of transcranial electric stimulation using a three layer head model. Engineering Analysis with Boundary Elements, 123, pp. 70-83, 2021.

[10] Smolyak, S., Quadrature and interpolation formulas for tensor products of certain classes of functions. Soviet Mathematics Doklady, 4, pp. 240-243, 1963.

[11] Barthelmann, V., Novak, E. \& Ritter, K., High dimensional polynomial interpolation on sparse grids. Advances in Computational Mathematics, 12(4), pp. 273-288, 2000.

[12] Nobile, F., Tempone, R. \& Webster, C.G., A sparse grid stochastic collocation method for partial differential equations with random input data. SIAM Journal on Numerical Analysis, 46(5), pp. 2309-2345, 2008.

[13] Stoyanov, M., User manual: TASMANIAN sparse grids. Technical Report ORNL/TM2015/596, Oak Ridge National Laboratory, Oak Ridge, TN, 2015.

[14] Stoyanov, M.K. \& Webster, C.G., A dynamically adaptive sparse grids method for quasi-optimal interpolation of multidimensional functions. Computers \& Mathematics with Applications, 71(11), pp. 2449-2465, 2016.

[15] ITIS, Tissue properties database, 2019.

[16] Bikson, M., Asif, R. \& Datta, A., Computational models of transcranial direct current stimulation. Clinical EEG and Neuroscience, 43(3), pp. 176-183, 2012. 